\title{
Erratum: Relativistic electron vortex beams in a constant magnetic field [Phys. Rev. A 95, 063812 (2017)]
}

\author{
A. Rajabi and J. Berakdar \\ (Received 17 September 2017; published 29 September 2017)
}

DOI: 10.1103/PhysRevA.96.039903

Equation (26) of the published article contains an error. $J_{n}^{l+2 s}$ for negative total angular momentum $m$ has to be changed to $J_{n+2 s}^{l+2 s}$. The correct expression for the spinor wave function in a uniform magnetic field in terms of the sign of $m$ reads

$$
\begin{aligned}
& \psi(\vec{r}, t)_{m>0}=C \delta_{s, 1 / 2}\left(\begin{array}{c}
\mathbf{J}_{n}^{l}\left(\frac{2 r^{2}}{\omega^{2}}\right) \\
0 \\
\frac{k_{z}}{E+m_{0}} \mathbf{J}_{n}^{l}\left(\frac{2 r^{2}}{\omega^{2}}\right) \\
\frac{2 i \sqrt{(2 n+|l|+1+l+2 s)}}{\omega\left(E+m_{0}\right)} \mathbf{J}_{n}^{l+2 s}\left(\frac{2 r^{2}}{\omega^{2}}\right)
\end{array}\right)+C \delta_{s,-(1 / 2)}\left(\begin{array}{c}
0 \\
\mathbf{J}_{n}^{l}\left(\frac{2 r^{2}}{\omega^{2}}\right) \\
-\frac{2 i \sqrt{(2 n+|l|+1+l+2 s)}}{\omega\left(E+m_{0}\right)} \mathbf{J}_{n}^{l+2 s}\left(\frac{2 r^{2}}{\omega^{2}}\right) \\
-\frac{k_{z}}{E+m_{0}} \mathbf{J}_{n}^{l}\left(\frac{2 r^{2}}{\omega^{2}}\right)
\end{array}\right), \\
& \psi(\vec{r}, t)_{m<0}=C \delta_{s, 1 / 2}\left(\begin{array}{c}
\mathbf{J}_{n}^{l}\left(\frac{2 r^{2}}{\omega^{2}}\right) \\
0 \\
\frac{k_{z}}{E+m_{0}} \mathbf{J}_{n}^{l}\left(\frac{2 r^{2}}{\omega^{2}}\right) \\
-\frac{2 i \sqrt{(2 n+|l|+1+l+2 s)}}{\omega\left(E+m_{0}\right)} \mathbf{J}_{n+2 s}^{l+2 s}\left(\frac{2 r^{2}}{\omega^{2}}\right)
\end{array}\right)+C \delta_{s,-(1 / 2)}\left(\begin{array}{c}
0 \\
\mathbf{J}_{n}^{l}\left(\frac{2 r^{2}}{\omega^{2}}\right) \\
\frac{2 i \sqrt{(2 n+|l|+1+l+2 s)}}{\omega\left(E+m_{0}\right)} \mathbf{J}_{n+2 s}^{l+2 s}\left(\frac{2 r^{2}}{\omega^{2}}\right) \\
-\frac{k_{z}}{E+m_{0}} \mathbf{J}_{n}^{l}\left(\frac{2 r^{2}}{\omega^{2}}\right)
\end{array}\right) .
\end{aligned}
$$

Accordingly the probability density and the azimuthal component of the current should read where (LG) represents the Laguerre-Gaussian beam,

$$
\begin{aligned}
& \rho^{\mathrm{LG}}(r)_{m>0}=\left(1-\frac{e B(2 n+|l|+|2 s|+l+2 s)}{2 E\left(E+m_{0}\right)}\right)\left|\mathbf{J}_{n}^{l}\left(\frac{2 r^{2}}{\omega^{2}}\right)\right|^{2}+\frac{e B(2 n+|l|+|2 s|+l+2 s)}{2 E\left(E+m_{0}\right)}\left|\mathbf{J}_{n}^{l+2 s}\left(\frac{2 r^{2}}{\omega^{2}}\right)\right|^{2}, \\
& \rho^{\mathrm{LG}}(r)_{m<0}=\left(1-\frac{e B(2 n+|l|+|2 s|+l+2 s)}{2 E\left(E+m_{0}\right)}\right)\left|\mathbf{J}_{n}^{l}\left(\frac{2 r^{2}}{\omega^{2}}\right)\right|^{2}+\frac{e B(2 n+|l|+|2 s|+l+2 s)}{2 E\left(E+m_{0}\right)}\left|\mathbf{J}_{n+2 s}^{l+2 s}\left(\frac{2 r^{2}}{\omega^{2}}\right)\right|^{2}, \\
& j_{\varphi}^{\mathrm{LG}}(r)_{m>0}=\frac{2 n !}{E \omega} \sqrt{\frac{(2 n+|l|+|2 s|+l+2 s)}{(n+|l|) !(n+|l+2 s|) !}} e^{-\left(2 r^{2} / \omega^{2}\right)}\left(\frac{\sqrt{2} r}{\omega}\right)^{|l|+|l+2 s|} \mathbf{L}_{n}^{|l|}\left(\frac{2 r^{2}}{\omega^{2}}\right) \mathbf{L}_{n}^{|l+2 s|}\left(\frac{2 r^{2}}{\omega^{2}}\right), \\
& j_{\varphi}^{\mathrm{LG}}(r)_{m<0}=-\frac{2}{E \omega} \sqrt{\frac{n !(n+2 s) !(2 n+|l|+|2 s|+l+2 s)}{(n+|l|) !(n+|l+2 s|) !}} e^{-\left(2 r^{2} / \omega^{2}\right)}\left(\frac{\sqrt{2} r}{\omega}\right)^{|l|+|l+2 s|} \mathbf{L}_{n}^{|l|}\left(\frac{2 r^{2}}{\omega^{2}}\right) \mathbf{L}_{n+2 s}^{|l+2 s|}\left(\frac{2 r^{2}}{\omega^{2}}\right) .
\end{aligned}
$$
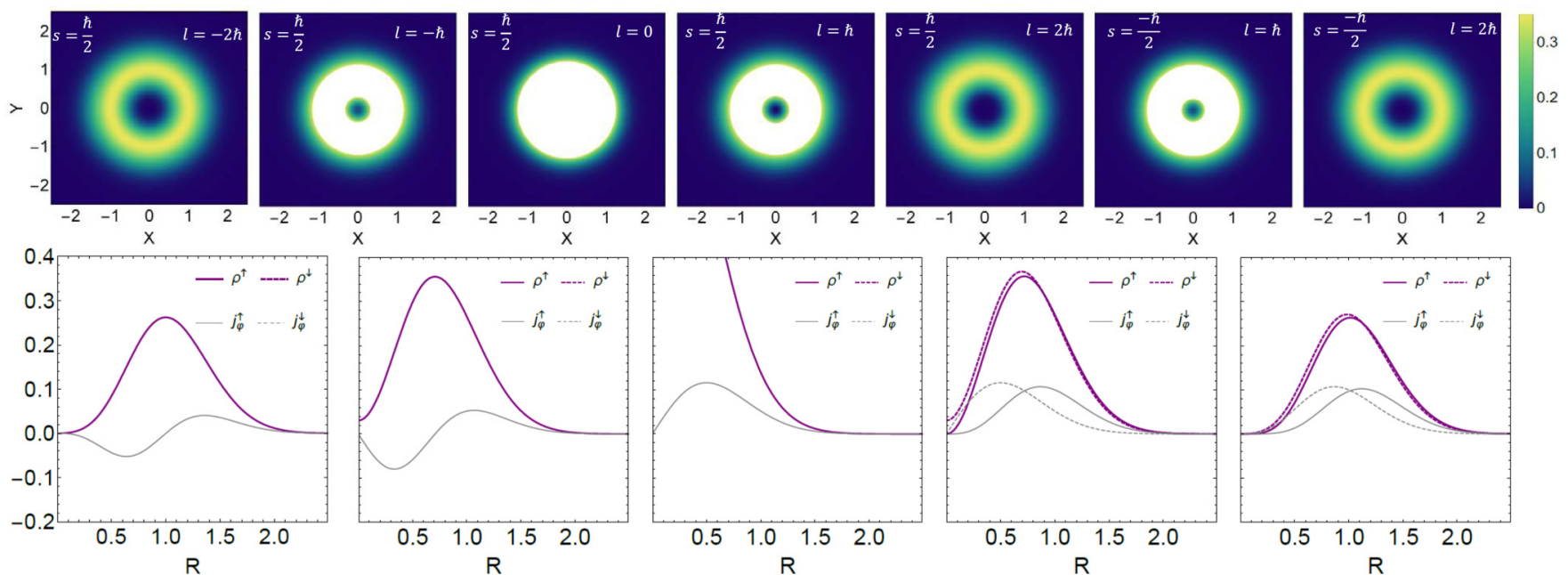

FIG. 1. This figure replaces Fig. 2 of the original paper. 

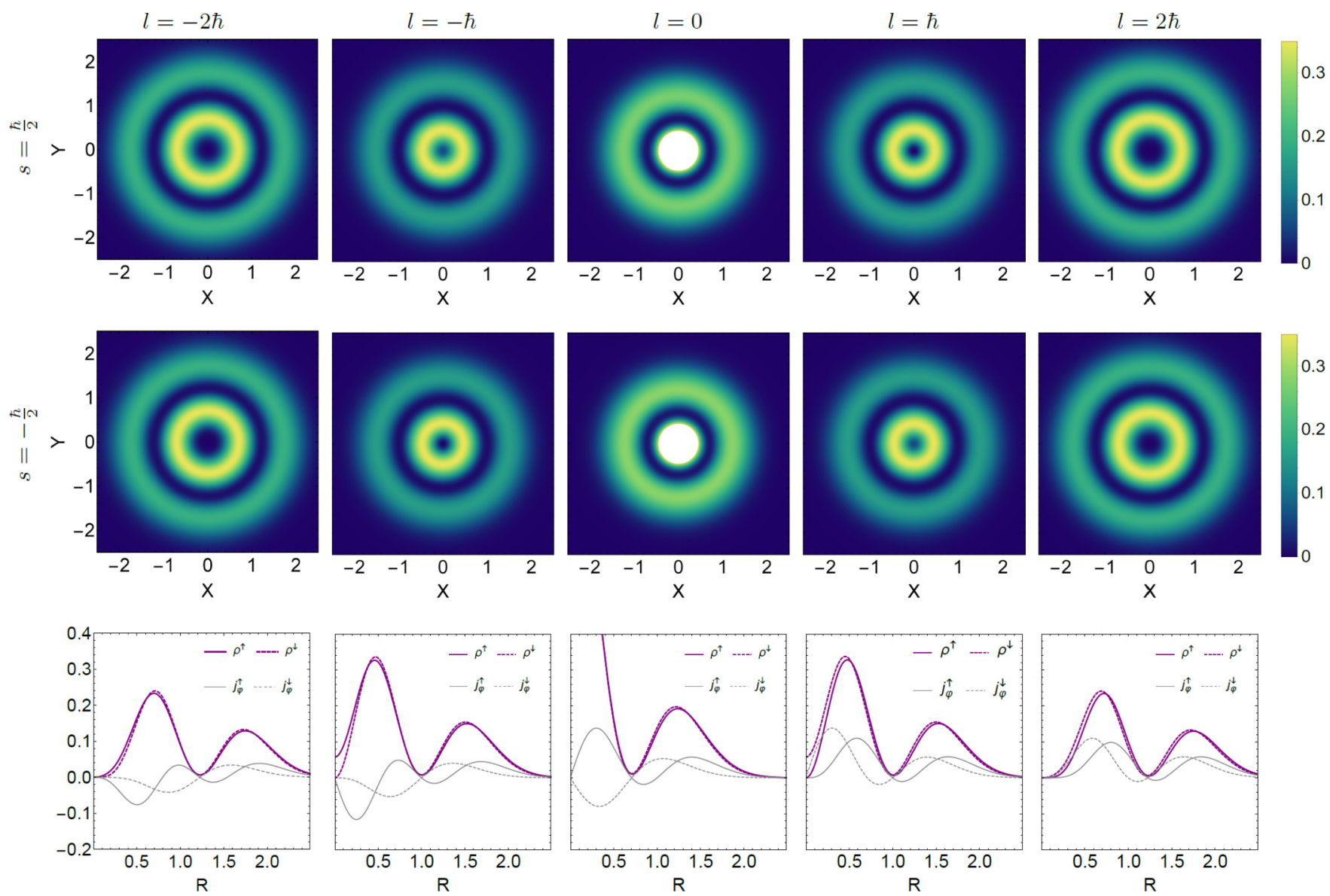

FIG. 2. This figure replaces Fig. 3 of the original paper.

For $m<0$ and $s=-\frac{1}{2}$, the radial quantum number $n$ starts from 1. The illustrations in Figs. 1 and 2 of the corrected equations replace Figs. 2 and 3 of the original paper.

We acknowledge K. van Kruining, J. Götte, and A. Hayrapetyan for pointing out the error in Eq. (26) of the original paper and for numerous communications. 